\title{
A Rare Case Report on Madura Foot
}

\author{
Vasavi Bysani*, Asha Latha Pabbathi, Divya Gopineni \\ Department of Pharmacy Practice, Sri Padmavathi School of Pharmacy, Tirupathi, Andhra Pradesh, INDIA.
}

\begin{abstract}
Mycetoma is a chronic infectious disease of the subcutaneous tissue. It is new designated by World Health Organization (WHO) which is endemic in tropical and subtropical regions. ${ }^{1}$ Mycetoma can be caused by a large variety of microorganisms. The infection caused by tramautic inoculation of a (Eumycetoma) Fungi or a (Actinomycetoma) bacterium. ${ }^{2}$ It is a disorder of subcutaneous tissue, skin and bones, mainly of feet, characterized by a traid of localized swelling underlying sinus tracts and production of granules. ${ }^{3}$ Treatment of disease depends on the etiology of the causative agent. ${ }^{2}$ Since the treatment of two etiologies is entirely different, A definite diagnosis after histopathological and microbiological examination is necessary, Serological test exists but is not so reliable. ${ }^{3}$ The treatment has been surgical debridment or amputation. Noninvasive management with long term antimicrobials has been successfully reported.
\end{abstract}

Key words: Actinomycetoma, Madurafoot, Mycetoma, Nocardia, Dapsone.

\section{INTRODUCTION}

Mycetoma is a devasting chronic subcutaneous granulamatous inflammatory disease caused by bacteria and fungi. Maduramycosis caused by fungi, Actinomycosis caused by actinmyces $\mathrm{k}$ which belonged to bacteria. The oldest description of the disease, Gill first recognized mycetoma as a disease entity in 1842 in the southeren province of Madura, The commonly used name "Madura Foot". Godfrey first documented a case of mycetoma in Madras, India. Mycetoma is reported worldwide "Mycetoma belt". Mycetoma is more commonly reported in males than females (3:1) because men being more commonly involved in agricultural work. The disease is charcterised by numerous deformations and disabilities, high morbidity and its late stage is potential fate. ${ }^{4}$ The most common site of occurrence is foot (approximately 70\% cases), Hand is the next common site. Infection can be cuased by fungi 40\% cases and 60\% cases by bacteria. ${ }^{5}$ No age is exempted from infection but the majority of patients are young adults in the age. Children account for $30 \%$ of the cases. ${ }^{6}$ In most of the reported cases, The disease is notoriously difficult to treat because the treatment consists of long course of antifungals and antibacterial often combined with surgery. Mycetoma patients are unique. They are of low socioeconomic status and health education level, residue in remote, poor rural areas with medical health resources. The introduction of new broadspectrum antimicrobials and antifungals improved the hope of drug efficiency.

\section{CASE REPORT}

A 30 years male patient was presented to the dermatology department with chief complaints of swelling of right leg for 3 years. patient was apparently normal 3 years back and then development of swelling on right leg which then started to discharge by then gradually progressed to involved foot and back of leg on period of 3 years. The biopsy and pus culture test was done 3 years back. He was a known smoker and alcoholic stopped 2 months back and takes mixed diet. On cutaneous examination, the swelling of size $10 * 8 \mathrm{~cm}$ present over posterior aspect of ankle irregular in shape, hyperpigmented skin
DOI: 10.5530/ijopp.12.1.11

Address for correspondence: Dr. Vasavi Bysani, Department of Pharmacy Practice, Sri Padmavathi School of Pharmacy, Jawaharlal Nehru Technological University, Anantapur,

Andhra Pradesh- 517503, INDIA. Phone no: +91 7207775633 Email Id: vvasavibysani@gmail. com

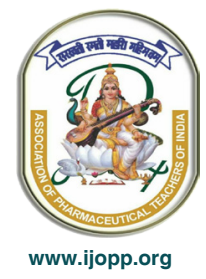


over swelling with multiple discharge sins us and pustules and also edema of right leg present with indication up to knee, pus discharge present over the anterior lateral and posterior aspect of right leg. On the examination the patient vital were stable. The total bilirubin ( $\mathrm{T}$ ) $0.9 \mathrm{mg} / \mathrm{dl}$ (D) $0.1 \mathrm{mg} / \mathrm{dl}$,(ID) $0.8 \mathrm{mg} / \mathrm{dl}$, SGOT (30U/L), SGPT(34IU/L), Sr. Cr (0.8)mg/dl were elevated. Then the culture sensitivity test was done and it was reported as staphylococcus aureus isolated and also AFB stain test was performed it was reported as negative.

\section{DISCUSSION}

Mycetoma is potentially serious devasting chronic inflammatory disease caused by actinomycetoma and eumycetoma. They are mainly caused by three genere (Nocardia, streptomyces, actinomadura). Most frequently causative agents of Nocardia include (Nocardia(N)) brasiliensis, N. asteroides. ${ }^{7}$ The etiologic agent of actinomadura includes (Actinomyces israeii etc). ${ }^{7}$

\section{Pathophysiology}

The causative organism enters through sites of local trauma eg; cut on the hand, thorn or foot splinter. A neutrophil response initially occurs, which may be followed by granulomatous reaction. Spread occurs through skin and can involve the bone.

\section{Clinical Presentation}

Over $75 \%$ of patients have a lesion of lower extremities. Most commonly affected in foot region (70\%) followed by hand other sites include the head, neck, chest, shoulder and arms. The incubation period from 3 months to 9 years. Most of the cases started as small painless, subcutaneous nodule at site of injury which over soften and ulcerate to discharge a viscous, purulent, serasanguinous fluid contain charcterstic granules. These granules composed of causative organism. The skin appears smooth shiny and commonly fixed to underlying tissue. skin may be hypo or hyper pigmented. ${ }^{8}$

\section{Treatment}

Actinomycetoma are more amenable to therapy than eumycetomas. Dapsone is oftenly used, since it is effective and expensive. Dapsone with streptomycin Trimethoprim-sulfamethoxazole (TMP-S) and (TMP-S) with Streptomycin or rifampicin are effective. Amikacin has been used in combination with (TMP-S) for recalcitrant thorax head lesions. other Antibiotics are minocycline, amoxiclav and imipenam are used in treatment of Madura foot. Ciprofloxacin has been effective in treating actinomycetomas with bone involvement. Response to therapy is good in 70\%-90\% with actinomycetomas even with bone involvement. Or sometimes debridement of the body part. ${ }^{9}$

\section{Clinical Message}

Mycetoma should be considered as a possible diagnosis, especially in tropical countries with barefoot walkers even in the absence of classical signs and should be treated after confirming with appropriate investigations.

\section{ACKNOWLEDGEMENT}

We would like to express our gratitude to all the physicians and PGs of Department of Dermatology, SVRRGGH for their support and cooperation.

\section{CONFLICT OF INTEREST}

The authors declare no conflict of interest.

\section{ABBREVIATIONS}

WHO: World Health Organisation; TMPs: Trimethoprim+sulphamethaxazole; AFB: Acid Fast Bacill; SGOT: Glutamic-0xalacetic Transaminase; SGPT: Glutamic-Pyruvic Transaminase.

\section{SUMMARY}

Mycetoma is chronic infection of skin and subcutaneous tissue. Mycetoma is caused by both fungi and bacteria Greater frequency of disease in men may not be just attributable to environmental factors but also to hormonal factors. Various serological and molecular tests have also found place in diagnosis of mycetoma. Combination antibiotic therapy is a must in case of actinomycetoma. Several newer antifungals have been tried for eumycetomas.

\section{REFERENCES}

1. Ahmed HF, Suliman HS, Roderick H. Mycetoma: The Spectrum of Clinical Presentation. Tropical Medicine of Infectious Disease. 2018;3(3):97.

2. Hala TM, Ahmed F, DeSande WWV. Mycetoma: epidemiology, treatment challenges and progress. Res Rep Tep Med. 2015;6:31-6.

3. Vineet R, Khushbu M, Pooja A. Mycetoma: An Update from Indian Journal of Dermatology. 2017.

4. Amit MS, Namitha S. Non-Invasive Management of Madura Foot with Oral Posaconazole and Ciprofloxacin. The American Journal of Tropical Medicine and Hygeine. 2014.

5. Kiran A, Veena M. Histological Diagnosis of Madura Foot: A Must for Definitive Treatment. Journal of Global Infectious Disease. 2009.

6. Hassan I, Keen A. Mycetoma Revisited from Nasza Dermatology Online. 2011.

7. Oliverio W, Al-Abdely HM. Mycetoma Medical Therapy from PLOS Neglected Tropical Disease. 2014.

8. Folusakin O, Ayoade MD. Mycetoma, From Medscape. 2017.

9. Robert B, Bs MT. (ASCP) Mycetoma An Overview, From CE Update (10Microbiology and Virology). 2010. 